\title{
Run-On Assays
}

National Cancer Institute

\section{Source}

National Cancer Institute. Run-On Assays. NCI Thesaurus. Code C19582.

A method to determine the constellation of sequences being actively transcribed at a given moment. Cell nuclei are harvested, and then supplied with a pulse of radioactive nucleotides. Transcription is allowed to proceed to an end, but additional initiations are inhibited. RNAs are then extracted and separated by gel electrophoresis. 